\title{
POTENCIAL DE LOS RESIDUOS FORESTALES PARA LA CONTRIBUCIÓN A LA MATRIZ ENERGÉTICA URBANA
}

\author{
POTENTIAL FROM FORESTRY WASTE FOR THE CONTRIBUTION TO THE URBAN \\ ENERGY MATRIX
}

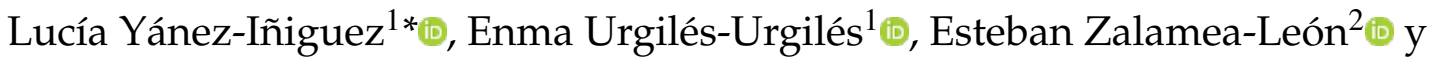 Antonio Barragán-Escandón ${ }^{3}$ (i)

\author{
${ }^{1}$ Facultad de Ciencias Químicas, Universidad de Cuenca. Av. 12 de Abril, 010107, Cuenca, Ecuador. \\ ${ }^{2}$ Facultad de Arquitectura y Urbanismo, Universidad de Cuenca. Av. 12 de Abril, 010107, Cuenca, Ecuador. \\ ${ }^{3}$ Grupo de Investigación de Energías (GIE), Universidad Politécnica Salesiana. Calle Vieja 12-30 y Elia Liut, 010105, Cuenca, \\ Ecuador. \\ *Autor para correspondencia: irina.yanezi@ucuenca.edu.ec
}

\begin{abstract}
Resumen
Los combustibles fósiles son por ahora la principal fuente de abastecimiento energético de las ciudades. Una estrategia para reducir este consumo es el desarrollo de energías renovables desde recursos endógenos urbanos. Se propone una metodología para determinar el potencial energético que poseen los residuos forestales urbanos en la ciudad de Cuenca-Ecuador, obtenidos mediante las actividades de mantenimiento (poda) de las áreas verdes públicas, con el propósito de transformarlos en fuente energética. Mediante análisis en laboratorio de muestras tomadas en el medio local, se determina que el poder calorífico inferior promedio que posee la biomasa es de 0.38 tep/ton. A partir de ello, con una base de datos estadísticos se calcula que en la ciudad de Cuenca se dispone de 608.63 ton de masa forestal anualmente. Ésta cuenta con un potencial energético de 233.13 tep/año y una eficiencia para la producción de energía eléctrica de aproximadamente 41 tep/año, que permite cubrir el consumo promedio de 110 familias. Se concluye que esta fuente de energía puede crecer significativamente con el incremento de las actividades de mantenimiento de las áreas verdes públicas y además constituye una estrategia para el aprovechamiento secundario de esta clase de residuos.
\end{abstract}

Palabras clave: Energías renovables, potencial energético, recursos endógenos, residuos forestales. 


\begin{abstract}
Nowadays, fossil fuels are the main source for energy supply in urban centers. Therefore, development of renewables from endogenous resources has become a strategy to reduce its consumption. Within that framework, this study case proposes a methodology to estimate the energy potential of urban forestry wastes in Cuenca-Ecuador city, which are obtained from maintenance activities at the public green areas, as an alternative energy source. It has been determined by laboratory analyses the average of the net calorific value of some biomass samples taken at the local area, and its result is about 0.38 tep/ton. From a statistical database, it has been calculated that forestry waste mass available per year in Cuenca city is 608.63 ton. Its energy potential is around 233.13 tep/year and the electrical generation efficiency is approximately $41 \mathrm{tep} /$ year, corresponding to the average consumption of 110 local families. Finally, it is concluded that this energy source could rise significantly through the increase of maintenance activities of public green areas. Furthermore, it represents an alternative for the effective use of this kind of waste.
\end{abstract}

Keywords: Renewable energies, energy potential, endogenous resources, forestry wastes.

$\begin{array}{ll}\text { Forma sugerida de citar: } & \begin{array}{l}\text { Yánez-Iñiguez, L., Urgilés-Urgilés, E., Zalamea-León, E. y Barragán-Escandón, A. (2020). Poten- } \\ \text { cial de los Residuos Forestales para la contribución a la Matriz Energética Urbana. La Granja: } \\ \text { Revista de Ciencias de la Vida. Vol. 32(2):42-53. http:/ /doi.org/10.17163/lgr.n32.2020.04. }\end{array}\end{array}$

IDs Orcid:

Lucía Yánez-Iñiguez: http:/ / orcid.org/0000-0002-1602-3464

Enma Urgilés-Urgilés: http:/ / orcid.org/0000-0001-5511-7570

Esteban Zalamea-León: http:/ / orcid.org/0000-0001-5551-5026

Antonio Barragán-Escandón: http:/ / orcid.org/0000-0003-2254-2524

La Granja: Revista de Ciencias de la Vida 32(2) 2020:42-53. 


\section{Introducción}

La diversificación de las fuentes de Energía Renovable (ER) es clave para contar con sistemas de abastecimiento sustentables, más aún cuando se pueden aprovechar los residuos urbanos (Arrese y Blanco, 2016). El autoabastecimiento energético desde recursos endógenos es esencial para reducir la necesidad de importación de energía en las ciudades (Barragán y col., 2019). La matriz energética en Cuenca-Ecuador, posee una alta dependencia de combustibles fósiles, cuyos métodos de extracción, procesamiento y transformación constituyen una importante problemática ambiental (Bristow y Kennedy, 2013). La biomasa forestal dentro de un contexto energético hace referencia al conjunto de elementos renovables de origen orgánico o sus derivados, cuya energía procede de la radiación solar que es transformada en energía química de enlace durante el proceso de fotosíntesis realizado por las especies vegetales (Manzano y col., 2012). Esta energía química puede ser utilizada directamente a partir de procesos de combustión o ser transformada mediante métodos térmicos (gasificación) o biológicos (producción de bioetanol), de acuerdo al requerimiento final de uso (Yaman, 2004).

Los residuos forestales que provienen de las operaciones de podas urbanas pueden ser aprovechados como una ER para la producción de electricidad a partir de procesos térmicos (Pérez, Borge y Agudelo, 2010). Una de las ventajas que posee la biomasa forestal residual es la carencia de un valor ecológico o agrícola, a diferencia de otros tipos de residuos vegetales no arbóreos (Barragán, 2018). El mantenimiento de las Áreas Verdes Públicas (AVP) es además una necesidad de adecentamiento urbano, que genera colateralmente una producción continua del recurso. En cinco ciudades de Corea del Sur (Seúl, Daegu, Daejaeon, Gwangju, Busan) se ha analizado el Potencial Energético (PE) de la biomasa procedente de los productos forestales urbanos, con el fin de establecer un manejo adecuado para su conversión a energía, obteniéndose capacidades máximas y mínimas de 2625753 tep/año y 76760 tep/año, respectivamente (Kook y Lee, 2015). En General Pueyrredón (Argentina), se ha evaluado la energía potencial aprovechable de las podas de los espacios públicos, determinando que éstas pueden satisfacer alrededor del $4.37 \%$ del consumo eléctrico que requiere la ciudad (Roberts y col., 2015). Sin embargo, cada localidad cuenta con condiciones propias, ya sea por la cantidad o por las características de la vegetación (Barragán, 2018). De la misma forma que acorde al tipo de consumo energético las metodologías de dimensionamiento y proporcionamiento también difieren, lo que significa gran variabilidad en cada una de las investigaciones de esta área.

Al analizar estos casos de estudio se ha establecido que para determinar el PE se requiere conocer el Poder Calorífico Inferior (PCI) de las especies que conforman los residuos forestales, la masa de producción anual y el porcentaje de eficiencia que tiene el recurso para la generación de energía. En la ciudad de Cuenca, la Empresa Pública Municipal de Aseo para Cuenca (EMAC EP), tiene a su cargo el mantenimiento de las AVP de la urbe, y que se encuentran distribuidas en tres categorías: parques, riberas y parterres (Ortiz, 2018), lo que convierte a estos espacios en fuentes para la obtención del recurso renovable mencionado.

\section{Materiales y métodos}

\subsection{Aspectos generales del área de estudio}

El área de estudio comprende las AVP: parques, parterres y riberas (Figura 1), del casco urbano de la ciudad de Cuenca, localizada en la provincia del Azuay en la región centro-sur del Ecuador. El centro urbano cuenta con una superficie de alrededor de $72 \mathrm{~km}^{2}$ y una población de 391657 habitantes (INEC, 2016). Cuenca se encuentra a una altura aproximada de 2550 m.s.n.m. y posee una temperatura promedio de $15,6^{\circ} \mathrm{C}$, que puede oscilar entre $27,2^{\circ} \mathrm{C}$ y $-1,7^{\circ} \mathrm{C}$ (Plan Estratégico Cuenca 2020, 2004).

Para la presente investigación se utilizó la información cartográfica correspondiente a las AVP establecidas dentro del Proyecto Cinturón Verde de la ciudad de Cuenca y se trabajó con imágenes satelitales con fecha 20 de abril del año 2018, que contaban con una resolución de $3,14 m \times 3,14 m$ por píxel y un porcentaje de nubosidad de $19 \%$. 


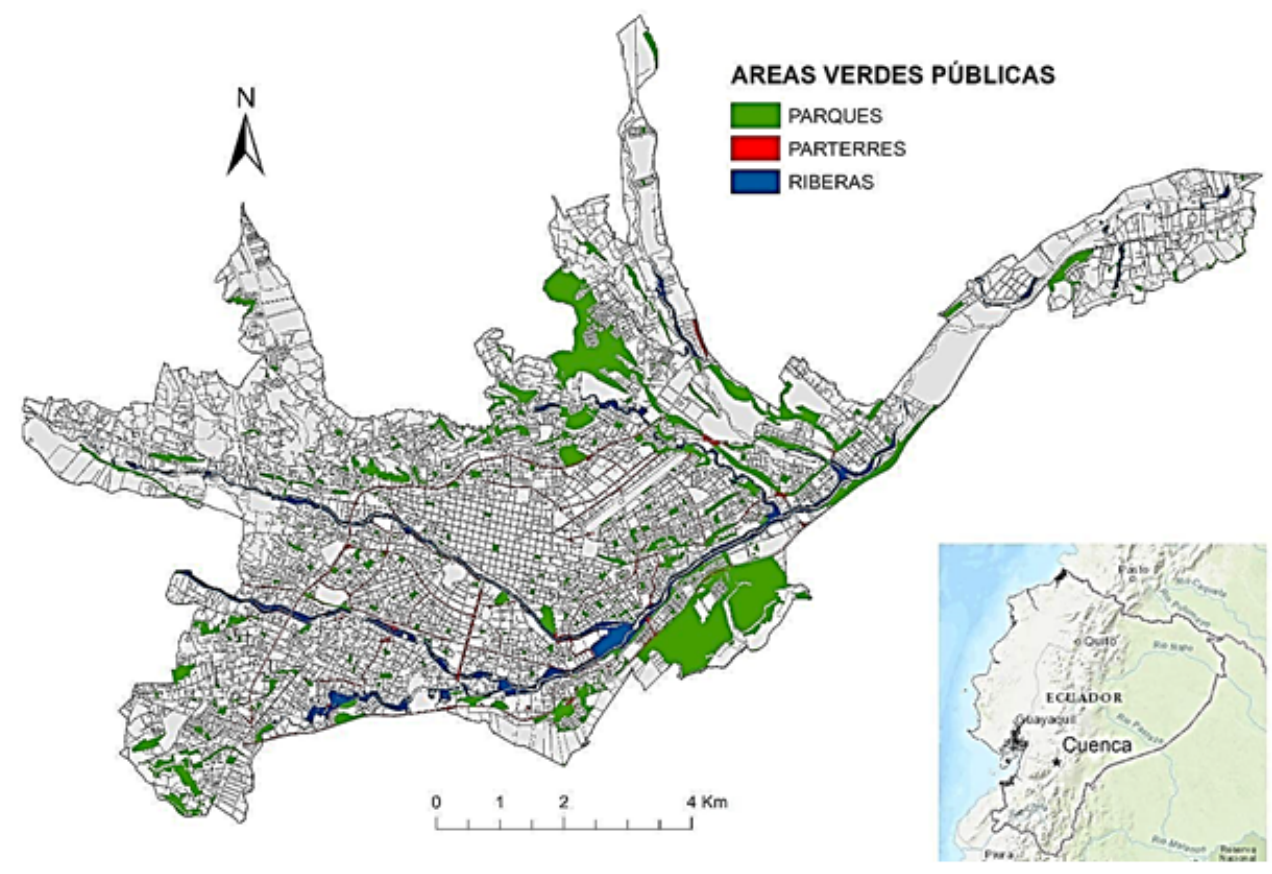

Figura 1. Área de estudio.

\subsection{Identificación de las especies forestales que forman parte de las podas de las AVP}

Para evaluar el PE de los residuos forestales se requiere conocer cuáles son las especies vegetales del área de estudio (Roberts y col., 2015). En este caso se consideró conveniente identificarlas directamente en las AVP que reciben mantenimiento de la Empresa Pública EMAC, debido a que las podas urbanas están constituidas por varios elementos forestales de pequeño tamaño, situación que dificulta el proceso para establecer a qué especie corresponde cada uno. De acuerdo a la metodología sugerida por Gutiérrez y col. (2015) se realizó un monitoreo durante el mes de agosto del año 2018 para la identificación de las especies. Para ello se escogieron los predios de las AVP que cumplían con dos criterios: i) una superficie $\geq 0,05$ ha $y \leq 1,21$ ha, $y$ ii) un Índice de Vegetación de Diferencia Normalizada $(\mathrm{IVDN}) \geq 0,26$.

El límite mínimo y máximo de superficie se obtuvo mediante la Ecuación 1 propuesta por Olsson (2005), que corresponde al índice de confiabilidad, para lo que se utilizó un nivel de confianza del $90 \%$. El valor de 0,26 representa el dato promedio del
IVDN, calculado para las AVP mediante el programa SIG ArcMap ${ }^{\circledR}$. Con $\chi$ (ha) la superficie media de las AVP, $\sigma$ (ha) es la desviación estándar de las áreas de los predios considerados como AVP, $n$ hace referencia al número total de AVP, $Z$ (\%) es el nivel de confianza y $\mu$ indica el número total de AVP encontradas dentro del intervalo de confianza (Olsson, 2005).

$$
\chi-\frac{Z * \sigma}{\sqrt{n}} \leq \mu \leq \chi+\frac{Z * \sigma}{\sqrt{n}}
$$

Este índice analiza mediante la Ecuación 2, sugerida por Rabatel, Gorretta y Labbé (2011), las características biofísicas de las plantas y la distribución vegetativa del área de estudio (Parthiban, Thummalu y Christy, 2015). Donde, IRC ( $\mu m)$ se refiere a la banda del Infra Rojo Cercano que poseen las imágenes satelitales y R $(\mu \mathrm{m})$ corresponde a la banda del Rojo (Rabatel, Gorretta y Labbé, 2011).

$$
I V D N=\frac{I R C-R}{I R C+R}
$$

El monitoreo de las especies vegetales distribuidas en las AVP (seleccionadas bajo los criterios descritos anteriormente) se efectuó en parcelas de 0,4 ha. Con la herramienta tecnológica ODK, que utiliza un servidor en línea para recopilar, administrar 
y almacenar información en un área determinada, se tomaron datos referentes a coordenadas de ubicación, foto, nombre común y nombre científico de los individuos encontrados.

\subsection{Obtención del poder calorífico de la biomasa forestal}

Para valorar energéticamente la biomasa forestal se considera adecuado analizar previamente su PCI. Este parámetro permite estimar la cantidad de energía aprovechable por unidad de masa cuando el recurso se combustiona completamente (Arroyo y Reina, 2017). El cálculo del PCI viene dado por la Ecuación 3, planteada por Francis y Lloyd (Budí, 2016). Donde, PCS ( $k c a l / k g)$ corresponde al valor del Poder Calorífico Superior, $597(\mathrm{kcal} / \mathrm{kg}$ ) es un indicador que hace referencia al calor procedente de la condensación del vapor de agua formado en el ensayo de combustión. El número 9 indica los $\mathrm{kg}$ de agua que se generan al oxidar $1 \mathrm{~kg}$ de hidrógeno, $H$ $(\%)$ es el porcentaje de cuantificación del hidrógeno $\mathrm{y} w(\%)$ se refiere al porcentaje de humedad que posee la biomasa (Budí, 2016).

$$
P C I=P C S-597 * 9 H+w
$$

Se identificaron 9 especies forestales como las más abundantes en las podas urbanas, que corresponden al 59,49\% de los individuos encontrados durante el monitoreo de campo. Se catalogaron en el estudio como especies representativas y se procedió a determinar su PCS y su porcentaje de humedad mediante análisis de laboratorio. La cuantificación del PCS se realizó en una bomba calorimétrica (IKA C200), y mediante los criterios de la Norma UNE-EN-ISO18135:2018 (Biocombustibles sólidos. Muestreo) se tomaron muestras compuestas por ramas y hojas de un individuo por cada una de las 9 especies (Ortiz T., 2013). Estos individuos fueron seleccionados de acuerdo a las características morfométricas de un ejemplar considerado como adulto (CONAFOR, 2013; Barahona, 2005; Biodiversidad (CONABIO), 2013; Minga y Verdugo, 2016).

Las muestras se acondicionaron de acuerdo a los parámetros establecidos por la Norma UNE-
CEN/TS 14918: 2011 (Biocombustibles sólidos. Determinación del poder calorífico), sometiéndolas a un proceso de secado a una temperatura de $105^{\circ} \mathrm{C} \pm$ 1 , durante 24 horas. Posteriormente, en la bomba calorimétrica se llevó a cabo el análisis del PCS, obteniendo un resultado digital dado en unidades de energía por masa. Tal como lo muestra el estudio de Vassilev y col. (2010), la biomasa forestal generalmente está compuesta por un $6 \%$ de hidrógeno, siendo éste el valor utilizado. El porcentaje de humedad para las especies representativas fue calculado con los criterios descritos en la Norma UNECEN/TS 14774: 2010 (Biocombustibles sólidos. Determinación del contenido de humedad. Método de secado en estufa), en donde se establece la Ecuación 4 para su cálculo. Donde, $m 1$ (g) es el peso de un crisol con la muestra antes del secado y $m 2$ (g) es el peso del mismo crisol con la muestra seca (UNECEN/TS 14774: 2010).

$$
w=\frac{m 1-m 2}{m 2} * 100
$$

\subsection{Estimación del potencial energético de los residuos forestales}

Para la estimación del PE (tep/año) se utilizó la Ecuación 5, que de acuerdo a lo planteado por Özdemir y Gencer (2016), permite relacionar el PCI (tep/ton) con la masa de los residuos forestales que se generan anualmente a partir de las operaciones de mantenimiento de las AVP. Ésta última se encuentra representada en la Ecuación 5 como $m$ (ton/año). El PCI corresponde al poder calorífico inferior promedio de las especies representativas, cuyas unidades fueron transformadas de $\mathrm{kcal} / \mathrm{kg}$ a tep/ton. Este valor se considera conservador puesto que la biomasa evaluada fue únicamente proveniente de especies forestales (leñosas), cuya composición orgánica es similar (celulosa, hemicelulosa y lignina) (Déjardin y col., 2010). La masa total de las podas se obtuvo mediante los datos estadísticos registrados en la Tabla 1 por el Departamento de Áreas Verdes de la Empresa Pública EMAC, durante los doce meses del año 2018.

$$
P E=P C I * m
$$


Tabla 1. Registro de podas urbanas durante 2018. Tomado de Emac (2018).

\begin{tabular}{cc}
\hline Mes & Masa (ton) \\
\hline Enero & 50.71 \\
Febrero & 50.72 \\
Marzo & 50.72 \\
Abril & 43.93 \\
Mayo & 66.08 \\
Junio & 58.35 \\
Julio & 47.52 \\
Agosto & 44.37 \\
Septiembre & 64.42 \\
Octubre & 41.01 \\
Noviembre & 64.95 \\
Diciembre & 25.81 \\
TOTAL & $\mathbf{6 0 8 . 6 3}$ \\
\hline
\end{tabular}

\subsection{Estimación de la eficiencia energética de los residuos forestales}

Debido a que los desechos forestales urbanos pueden ser aprovechados al convertirlos en electricidad mediante procesos térmicos (Barragán, 2018), como un primer acercamiento a la valoración energética de este recurso en la ciudad de Cuenca, se ha estimado la eficiencia energética con la Ecuación 6 (Panepinto, Viggiano y Genon, 2014), considerando el $18 \%$ como el porcentaje de rendimiento de la biomasa forestal para la conversión a energía eléctrica.

Este porcentaje permite establecer la energía efectivamente utilizable y hace referencia a la eficiencia para convertir la energía química de las especies vegetales a electricidad; factor referencial que se ha tomado a partir de un estudio desarrollado en la región de Basilicata-Italia, en donde se ha evaluado a las podas forestales como fuente de ER a través de un balance energético, el cual permite estimar la producción eléctrica mediante procesos térmicos. En la investigación Panepinto, Viggiano y Genon (2014), utilizan una metodología similar al presente caso y establecen como hipótesis que, en función de un escenario que cuenta con datos de la potencia energética disponible (calculada con la relación entre la cantidad de biomasa y el PCI de la misma), es posible valorar la eficiencia para la obtención de electricidad en un $18 \%$.

Por otra parte, existen metodologías que difieren y que utilizan eficiencias para la producción de electricidad de $10 \%$ (Shi y col., 2013) y de $4.37 \%$ (Ro- berts y col., 2015). Sin embargo, al ser éste un primer análisis del potencial energético de las podas urbanas en la localidad se ha visto pertinente utilizar el $18 \%$ como dato referencial. Para casos específicos se requiere un estudio con mayor profundidad que considere, entre otras variables, la eficiencia de los distintos dispositivos de conversión de energía.

$$
E E=P E * E
$$

Donde, $E E$ (tep/año) hace referencia a la energía producida en un año, $P E$ (tep/año) es el Potencial Energético de la biomasa forestal y $E(\%)$ corresponde al porcentaje de eficiencia para la generación de electricidad a partir del recurso (Panepinto, Viggiano y Genon, 2014).

\section{Resultados y Discusión}

\subsection{Especies forestales que forman parte de las podas de las AVP}

De acuerdo al muestreo desarrollado en las AVP de Cuenca, se identificó que las podas urbanas están conformadas por 72 especies forestales en total. La Tabla 2 muestra las especies encontradas que se ordenan en forma descendente con respecto a la abundancia. Para los fines del estudio se estableció que el $59,49 \%$ de los individuos contabilizados corresponden a 9 especies catalogadas como representativas (Eucalyptus globulus, Salix humboldtiana, Prunus serótina, Tecoma stans, Baccharis latifolia, Fraxinus excelsior, Callistemon salignus, Pinus radiata, Acacia dealbata), esto debido a que son las más abundantes. 
Tabla 2. Especies forestales identificadas en las áreas verdes públicas.

\begin{tabular}{|c|c|c|c|}
\hline Nombre Científico & Abundancia & Nombre Científico & Abundancia \\
\hline Eucalyptus globulus & 459 & Tipauna tipu & 7 \\
\hline Salix humboldtiana & 80 & Acacia baileyana & 6 \\
\hline Prunus serótina & 47 & Ferreyranthus verbascifolius & 6 \\
\hline Tecoma stans & 47 & Rubus glaucus & 6 \\
\hline Baccharis latifolia & 45 & Morella sp. & 6 \\
\hline Fraxinus excelsior & 43 & Yucca guatemalensis & 6 \\
\hline Callistemon salignus & 41 & Ficus Robusta & 4 \\
\hline Pinus radiata & 37 & Eucalyptus citriodora & 4 \\
\hline Acacia dealbata & 35 & Cotoneaster acuminatus & 4 \\
\hline Jacaranda mimosifolia & 32 & Liabum floribundum & 4 \\
\hline Schinus molle & 30 & Buddleja davidii & 3 \\
\hline Chionanthus pubescens & 29 & Laurus nobilis & 3 \\
\hline Sambucus mexicana & 27 & Monnina ligustrina & 3 \\
\hline Hibiscus rosa-sinensis & 26 & Citrus $x$ sinensis & 3 \\
\hline Juglans neotropica & 26 & Arecaceae & 3 \\
\hline Alnus acuminata & 25 & Myrcianthes hallii & 2 \\
\hline Cupressus lusitánica & 24 & Buxus sínica & 2 \\
\hline Podocarpus sprucei & 22 & Bougainvillea spectabilis & 2 \\
\hline Populus alba & 21 & Annona cherimola & 2 \\
\hline Morella pubescens & 21 & Cestrum nocturnum & 2 \\
\hline Syzygium paniculatum & 17 & Brugmansia sanguínea & 2 \\
\hline Inga insignis & 17 & Psidium guajava & 2 \\
\hline Acacia retinodes & 16 & Lantana cámara & 2 \\
\hline Ambrosia arborescens & 16 & Fuchsia boliviana & 2 \\
\hline Ficus benjamina & 14 & Robinia Pseudoacacia & 1 \\
\hline Eriobotrya japónica & 14 & Acalypha australis & 1 \\
\hline Ligustrum Japonicum & 13 & Populus balsamífera & 1 \\
\hline Acacia melanoxylon & 10 & Prunus persica & 1 \\
\hline Myrsine guianensis & 10 & Mimosa andina & 1 \\
\hline Jasminum polyanthum & 10 & Ligustrum sinense & 1 \\
\hline Callistemon citrinus & 9 & Crataegus pubescens & 1 \\
\hline Duhaldea cappa & 9 & Oreopanax ecuadorensis & 1 \\
\hline Erythrina edulis & 8 & Buddleja americana & 1 \\
\hline Nerium oleander & 8 & Rosmarinus officinalis & 1 \\
\hline Grevillea robusta & 8 & Rosa gallica & 1 \\
\hline Delostoma integrifolium & 7 & Citharexylum ilicifolium & 1 \\
\hline Delostoma integrifolium & 7 & & \\
\hline
\end{tabular}

\subsection{Poder calorífico de la biomasa forestal}

A partir de las evaluaciones realizadas en laboratorio se determinó el PCS de la biomasa proveniente de las especies representativas (Tabla 3). Al incorporar estos valores en el cálculo del PCI, se logró definir que la especie Pinus radiata es la que posee mayor capacidad para desprender calor durante los procesos de combustión completa, constituyéndose en el recurso más eficiente para la obtención de energía. A ésta le siguen Callistemon salignus y Prunus serótina. Esta última es considerada como una de las especies nativas de la ciudad de Cuenca a la que se recomienda para ser incorporada en los planes de reforestación manejados por la Empresa Pública EMAC, ya que se trata de una alternativa adecuada para la utilización de las AVP como fuente de recursos energéticos.

El estudio establece que es preferible emplear los productos de las podas de las AVP ya existentes para un aprovechamiento de la biomasa enfocado a la generación de energía en un escenario sostenible. Es decir, no se plantea la siembra de especies vegetales con fines energéticos, sino el uso de las mismas 
con criterio ambiental, ornamental y paisajístico.

Mediante los datos presentados en la Tabla 3, se definió que el PCI promedio de las especies forestales distribuidas en las AVP es de 0,38 tep/ton. Cabe mencionar que para obtener este valor se consideró adecuado evaluar el PCI únicamente de las 9 especies más abundantes $(59,49 \%)$, debido a la restricción del número de muestras a ser analizadas para el estudio, ya que por la diferencia del conte- nido de humedad (Tabla 3), cada una requiere de un análisis por separado y un número mínimo de tres pruebas. Además, al haber trabajado solamente con especies forestales cuya composición orgánica es similar (Déjardin y col., 2010), es posible establecer un valor promedio. El resultado obtenido $(0,38$ tep/ton) es cercano al determinado por Panepinto, Viggiano y Genon (2014), quienes muestran en su investigación que los productos forestales cuentan con un PCI de 0.34 tep/ton.

Tabla 3. Poder calorífico de las especies representativas.

\begin{tabular}{cccc}
\hline Nombre científico & $\begin{array}{c}\text { PCS } \\
\text { (tep/ton) }\end{array}$ & $\begin{array}{c}\text { Contenido de Humedad } \\
(\mathbf{\%})\end{array}$ & $\begin{array}{c}\text { PCI } \\
\text { (tep/ton) }\end{array}$ \\
\hline Eucalyptus globulus & 0.41 & 16.9 & 0.37 \\
Salix humboldtiana & 0.43 & 61.1 & 0.36 \\
Prunus seótina & 0.46 & 51.65 & 0.4 \\
Tecoma stans & 0.43 & 46.2 & 0.37 \\
Baccharis latifolia & 0.43 & 61.25 & 0.36 \\
Fraxinus excelsior & 0.42 & 45.02 & 0.42 \\
Callistemon salignus & 0.48 & 46.53 & 0.43 \\
Pinus radiata & 0.5 & 49.46 & 0.39 \\
Acacia dealbata & 0.45 & 41.22 & 0.3 \\
\hline PROMEDIO & & & $\mathbf{0 . 3 8}$ \\
\hline
\end{tabular}

\subsection{Potencial energético de los residuos fo- restales}

Mediante la Ecuación 5, que establece la relación entre la cantidad de biomasa disponible durante un año y su energía aprovechable correspondiente al PCI, se determinó que el PE de los residuos forestales es de aproximadamente 233,13 tep/año. Esta energía proviene de las podas que realiza actualmente la Empresa Pública EMAC en las áreas públicas que poseen biomasa forestal, que ocupan 618,76 ha de la superficie total de la ciudad de Cuenca.

La biomasa obtenida a partir de las operaciones de mantenimiento de las AVP puede convertirse en un recurso renovable alternativo para suplir las necesidades energéticas de la ciudad, tal y como lo plantean Kook y Lee (2015), quienes en un estudio desarrollado en centros urbanos de Corea del Sur han determinado que los residuos forestales cuentan con un potencial mínimo de 76760 tep/año. Esta diferencia de valores identificada entre las ciudades asiáticas y la zona urbana de Cuenca se debe en gran medida a dos factores principales: primero al tamaño de las ciudades, de lo que dependerá la superficie disponible para extraer el recurso y luego el uso final que tienen las podas actualmente en cada lugar. Las ciudades de Corea del Sur se catalogan como grandes metrópolis que disponen de espacios verdes que producen abundante biomasa (Kook y Lee, 2015), mientras que Cuenca cuenta con 7200 ha de superficie en total. Por otra parte, la localidad carece de un plan de manejo integral de residuos forestales, situación que difiere de las ciudades coreanas, en donde se ha reconocido la importancia real de las podas urbanas como recurso para la generación de ER.

La investigación desarrollada por Kook y Lee (2015) evidencia algunas limitaciones a ser tomadas en cuenta para integrar a la biomasa forestal como fuente de energía en las urbes; por ejemplo, el incremento acelerado y continuo de ocupación espacial en las ciudades, cuya área destinada para las edificaciones tiende a incorporar mayor espacio frente a las superficies de áreas verdes (Franco, 2012), así como el aumento del número de habitantes y con 
esto el incremento de las necesidades energéticas (Lahoz, 2010). Factores que deberán ser analizados previamente con el fin de evitar la sobreexplotación y el manejo no sostenible de la biomasa, producto de las podas.

Desde la perspectiva del uso de fuentes diversificadas, los residuos forestales son un recurso endógeno disponible que puede complementarse con energías renovables intermitentes como la solar y la eólica (Brown y col., 2018). Para el caso de Cuenca, una alternativa adecuada es la conversión de la biomasa a electricidad, considerando la mínima demanda térmica existente debido al clima, a diferencia de otras latitudes en las que la necesidad de ambientación espacial es preponderante.

\subsection{Eficiencia energética estimada para los residuos forestales}

La ciudad de Cuenca presenta una demanda total de energía eléctrica de $423800 \mathrm{MWh}$ /año (Barragán, 2018), de la cual actualmente el $38 \%$ corresponde al sector residencial. En este marco, al evaluar los residuos forestales como un recurso que posee el $18 \%$ de eficiencia energética, se obtuvo como resultado que la generación eléctrica a partir del mismo será de aproximadamente 476,83 MWh/año (41 tep/año). Producción con la cual es posible abastecer alrededor del 0,30\% de la demanda citada sobre el sector residencial, lo que representa la cobertura en la ciudad para 110 familias típicas de cuatro integrantes (familias promedio), considerando que cada uno de estos hogares posee un consumo total anual de 4,33 MWh/habitante (Barragán, 2018).

Hoy en día Cuenca cuenta con un modelo de ER que utiliza los desechos sólidos domésticos que llegan al relleno sanitario para el funcionamiento de una planta generadora de biogás, mediante la que se producen 502,60 tep/año de electricidad (Barragán, Arias y Terrados, 2016). Estos niveles de generación energética son mayores con respecto a los obtenidos a partir de los residuos forestales, y se debe a un manejo ya implementado para la recuperación secundaria de los desechos domésticos mencionados.

La energía procedente de fuentes renovables se podría incrementar a medida que se desarrollen e incorporen tecnologías destinadas al aprovecha- miento de los residuos forestales como recurso energético, al igual que se ha realizado en países como Corea del Sur, constituyéndose éste en un escenario alentador al considerar que la biomasa forestal permite fortalecer un modelo de diversificación de la matriz energética, en la que los recursos endógenos pueden tener un valor agregado (Barragán, 2018). El uso de biomasa residual en sistemas bioenergéticos requerirá de un análisis previo de costos para el transporte de las podas urbanas hacia los centros de generación eléctrica, que pueden ser disminuidos mediante una valoración de la densidad energética espacial existente en cada localidad (Kook y Lee, 2015). También se deberá tomar en cuenta el valor que representa el proceso industrial de conversión a energía y el de los sistemas para la distribución de electricidad a los usuarios (Yemshanov y col., 2014); para entonces analizar la real capacidad del recurso.

\section{Conclusiones}

Se presenta una metodología para determinar el potencial energético de los residuos forestales urbanos aplicable a cualquier urbe. La evaluación de la biomasa forestal con fines de producción eléctrica representa una alternativa para el manejo de las podas provenientes de las AVP que pertenecen a la zona urbana de la ciudad de Cuenca, Ecuador.

La información referente a la cantidad de energía por unidad de masa (PCI) que poseen las especies estudiadas permite establecer cuáles permiten reforestar de forma prioritaria a la ciudad, considerando a la especie Prunus serotina como recomendable para esta actividad, debido a que se ha catalogado dentro de las especies nativas, lo que permitirá la conservación del ornato. En este marco la propuesta de reforestación de las AVP ya existentes con especies de alto valor energético, también genera la posibilidad de cumplir con los parámetros mínimos de superficie de área verde por habitante establecidos por la Organización Mundial de la Salud (OMS), así como posibilita el aumento de la participación de las energías renovables en la matriz energética local.

Anualmente en la ciudad de Cuenca se producen 608,63 ton de residuos forestales a partir de las operaciones de mantenimiento de las AVP, que tiene a su cargo la Empresa Pública EMAC. En este 
sentido, la recuperación secundaria (producción de energía) también representará un modelo de gestión circular para las podas urbanas, otorgándoles un valor agregado.

Mediante el presente estudio se concluye que en la ciudad de Cuenca el potencial energético de los residuos forestales se encuentra valorado en 233,13 tep/año, lo que representa aproximadamente 41 tep/año (476.83 MWh/año) de generación eléctrica. Esta producción es marginal respecto al consumo de electricidad local, alcanzando a abastecer las necesidades de 110 familias promedio. No obstante, los niveles de poda locales son reducidos comparados con otras zonas urbanas en las que se realiza un trabajo paisajístico, debido a que se cuenta únicamente con una programación de actividades para el mantenimiento de aquellas AVP en donde es estrictamente necesario, o en casos en los que las especies forestales se encuentran interfiriendo con los cables de red del alumbrado público. Por lo tanto, si se establece una planificación para el manejo continuo de las AVP que contemple el aumento de las podas, se favorecerá a la obtención del recurso para fines energéticos, a la vez que será posible optimizar su recolección. Además, la cantidad del recurso podría incrementarse si se incorpora en el análisis la gestión de residuos privados, no dimensionados.

Se plantea como necesidad futura la evaluación técnica y económica para identificar tecnologías eficientes y rentables que se acoplen a las características del recurso natural evaluado, así como a los requerimientos de abastecimiento eléctrico que existen en la localidad. Estos análisis deberán contar con un enfoque de manejo sostenible, con el fin de evitar riesgos de sobreexplotación de la biomasa forestal. Además, son un complemento con las tecnologías renovables intermitentes, al poder introducir a la red la energía generada en horas pico.

Los residuos forestales procedentes de las podas de la ciudad forman parte de las alternativas para la diversificación de los recursos que constituyen la matriz energética a nivel local y nacional. Por lo que su evaluación, en el marco de las energías renovables, permite fortalecer un modelo de autoabastecimiento consecuente con la necesidad de disminuir la dependencia de los combustibles fósiles.

\section{Agradecimientos}

Este trabajo fue financiado por el Centro de Investigación de la Facultad de Arquitectura y Urbanismo de la Universidad de Cuenca y por la Dirección de Investigación de la Universidad de Cuenca DIUC, y forma parte del proyecto de investigación "Calibración de modelo F-Chart para colectores solares térmicos con parametrización y validación acorde a disposiciones típicas para integración arquitectónica en climas ecuatoriales andinos" $\left(\mathrm{N}^{\circ}\right.$ del Proyecto: 2040000 72146).

\section{Referencias}

Arrese, M. y G. Blanco (2016). «Territorio y energías renovables no convencionales: aprendizajes para la construcción de política pública a partir del caso de Rukatayo Alto, Región de Los Ríos, Chile». En: Gestión y política pública 25.1, 165-202. Online:https://bit.ly/2PrhbdS.

Arroyo, J. y W. Reina (2017). «Aprovechamiento del recurso biomasa a partir de los desechos de madera para una caldera de vapor». En: Ingenius, 20. Online:https:/ / bit.ly/2DEvdGk.

Barahona, L (2005). «Variación de la composición química en albura duramen y altura de madera pulpable de Eucalyptus globulus proveniente de monte alto y monte bajo». Tesis de mtría. Universidad de Chile.

Barragán, A. (2018). «El autoabastecimiento energético en los países en vías de desarrollo en el marco del metabolismo urbano: caso Cuenca, Ecuador». Tesis de mtría. Universidad de Jaén.

Barragán, E., P. Arias y J. Terrados (2016). «Fomento del metabolismo energético circular mediante generación eléctrica proveniente de rellenos sanitarios: estudio de caso, Cuenca, Ecuador». En: Ingenius 16, 36-42. Online:.

Barragán, E. y col. (2019). «Las energías renovables a escala urbana. Aspectos determinantes y selección tecnológica». En: Bitácora Urbano Territorial 29.2, 39-48. Online:https: / / bit.ly/2DbkvHP.

Biodiversidad (CONABIO), Comisión Nacional para el Conocimiento y Uso de la (2013). Fraxinus uhdei (Wenz.) Lingelsh. URL: https : / / bit. ly / 2ELgbPK.

Bristow, D. y C. Kennedy (2013). «Urban metabolism and the energy stored in cities: Implications for resilience». En: Journal of Industrial Ecology 17.5, 656-667. Online:https:// bit.ly/3fslssb. 
Brown, T. y col. (2018). «Response to 'Burden of proof: A comprehensive review of the feasibility of $100 \%$ renewable-electricity systems'». En: Renewable and Sustainable Energy Reviews 92, 834-847. Online:https://bit.ly/33sOJk4.

Budí, A. (2016). «Estimación del potencial energético de la biomasa residual agrícola y análisis de aprovechamiento en los municipios de la comarca del Alto Palancia». Tesis de mtría. Universitat Jaume I.

CONAFOR (2013). Fichas técnicas sobre características tecnológicas y usos de maderas comercializadas en México. URL: https:/ / bit.ly/2EOJH7f.

Déjardin, A. y col. (2010). «Wood formation in Angiosperms». En: Comptes rendus biologies 333.4, 325-334. Online:https://bit.ly/39VAA04.

Emac (2018). Empresa Municipal De Aseo De Cuenca. Municipio de Cuenca.

Franco, M. (2012). Análisis de los cambios en la cobertura y funcionalidad de áreas verdes en la Zona Metropolitana de la Ciudad de Mérida ( ZMM ). Inf. téc.

Gutiérrez, A. y col. (2015). «Parcela permanente de monitoreo de bosque de galería, en Puerto Gaitán, Meta». En: Corpoica. Ciencia y Tecnología Agropecuaria 16.1, 113-129. Online:https: / / bit . ly/2DAB4g9.

INEC (2016). Proyecciones poblacionales, Proyección de la Población Ecuatoriana, por años calenario, según cantones 2010-2020. Inf. téc. Instituto Nacional de Estadísticas y Censos.

Kook, J. W. y S. H. Lee (2015). "Analysis of biomass energy potential around major cities in South Korea». En: Applied Chemistry for Engineering 26.2, 178-183. Online:https: / / bit.ly / 2Xvqwpx.

Lahoz, E. (2010). «Reflexiones medioambientales de la expansión urbana». En: Cuadernos geográficos 46.46, 293-313. Online:https: / / bit.ly/3fs0WYQ.

Manzano, F. y col. (2012). «Insects for biodiesel production». En: Renewable and Sustainable Energy Reviews 16.6, 3744-3753. Online:https: / / bit.ly / 3icZ08n.

Minga, D. y A. Verdugo (2016). Árboles y arbustos de los ríos de Cuenca. Vol. Online: https: / / bit.ly / 2DoEt1M. Cuenca: Universidad del Azuay.

Olsson, U. (2005). "Confidence intervals for the mean of a log-normal distribution». En: Journal of Statistics Education 13.1. Online:https: / / bit. ly/3ftC570.
Ortiz T., L (2013). Estudio de caracterización de las biomasas forestales de interés energético existentes en el sur de Galicia y norte de Portugal. Tesis de mtría. Online: https: / / bit.ly /33xlLiX: Universidad de Vigo.

Ortiz, P. (2018). «Plan de acción territorial para la implantación de infraestructura verde en la ciudad de Cuenca». Tesis de mtría. Universidad de Cuenca.

Özdemir, Z. y A. Gencer (2016). «Determination of the Biomass Potential in Kirklareli Province Based on Agricultural Residues». En: 8TH EGE ENERGY SYMPOSIUM AND EXHIBITION, At Afyonkarahisar, TURKEY. Ed. por I. Dincer, C.O. Colpan y F. Kadioglu. New York. doi: 10.1007/978-1-4614-7588-0.: Springer.

Panepinto, D., F. Viggiano y G. Genon (2014). «The potential of biomass supply for energetic utilization in a small Italian region: Basilicata». En: Clean Technologies and Environmental Policy 16.5, 833-845. Online:https://bit.ly/2ELuAvi.

Parthiban, S., N. Thummalu y A. Christy (2015). «Ndvi: Vegetation change detection using remote sensing and gis - A case study of Vellore District». En: Procedia Computer Science 57, 1199-1210. Online:https://bit.ly/2XsERmJ.

Pérez, J., D. Borge y J. Agudelo (2010). «Proceso de gasificación de biomasa: una revisión de estudios teórico-experimentales». En: Revista facultad de ingeniería Universidad de Antioquia 52, 95-107. Online:https:/ /bit.ly/2BZQ46F.

Plan Estratégico Cuenca 2020 (2004). Ed. por Comité Ejecutivo. Municipio de Cuenca. Online:https: / / bit.ly/33tA7kA.

Rabatel, G., N. Gorretta y S. Labbé (2011). «Getting NDVI spectral bands from a single standard RGB digital camera: a methodological approach». En: Conference of the Spanish Association for Artificial Intelligence, 333-342. Online:https:/ / bit.ly/2Pph9mP.

Roberts, J. y col. (2015). «Assessment of dry residual biomass potential for use as alternative energy source in the party of General Pueyrredón, Argentina». En: Renewable and Sustainable Energy Reviews 41, 568-583. Online:https: / / bit. ly / 30rHxCQ.

Shi, Y. y col. (2013). «Garden waste biomass for renewable and sustainable energy production in China: Potential, challenges and development». En: Renewable and Sustainable Energy Reviews 22, 432-437. Online:https:/ / bit.ly/3fvV3cS. 
Vassilev, S. y col. (2010). «An overview of the chemical composition of biomass». En: Fuel 89.5, 913-933. Online:https:/ / bit.ly/30ttf4L.

Yaman, S. (2004). «Pyrolysis of biomass to produce fuels and chemical feedstocks». En: Energy conversion and management 45.5, 651-671. Online:https://bit.ly/2EIDSYX.

Yemshanov, D. y col. (2014). «Cost estimates of post harvest forest biomass supply for Canada». En: Biomass and Bioenergy 69, 80-94. Online:https: / / bit.ly/3fzOiZj. 